\title{
Perfil e atuação do agente comunitário de saúde no contexto da Estratégia Saúde da Família em dois municípios da Paraíba
}

\author{
Profile and role of community health workers in the context of the Family \\ Health Strategy in two municipalities of Paraíba
Profil et performance de l'agent de santé communautaire dans le cadre de la Stratégie de Santé Familiale dans deux municipalités de Paraíba

\author{
Perfil y actuación del agente comunitario de salud en el contexto de la \\ Estrategia Salud de la Familia en dos municipios de Paraíba
}

\author{
Dixis Figueroa Pedraza ${ }^{1}$ \\ Iná Santos ${ }^{2}$
}

Recebido em 22/02/2017; revisado e aprovado em 30/04/2017; aceito em 30/04/2017

DOI: http://dx.doi.org/10.20435/inter.v18i3.1507

\begin{abstract}
Resumo: Objetivou-se descrever o perfil e a realidade de trabalho do Agente Comunitário de Saúde (ACS) em dois municípios da Paraíba. Estudo transversal com 98 ACS. A maioria dos ACS desenvolvia as atividades estabelecidas para a profissão e trabalho burocrático. Agentes do município com menor dedicação à burocracia referiram maior atuação em mobilização comunitária e orientações sobre a saúde da criança. O desempenho do ACS pode ser prejudicado pelo envolvimento com tarefas burocráticas.

Palavras-chave: atenção primária à saúde; desenvolvimento da comunidade; Agentes Comunitários de Saúde.

Abstract : This study aimed to describe the profile and the reality of the Community Health Agent (ACS) work in two cities of Paraíba, Brazil. This was a cross-sectional study with 98 ACS. Most ACS developed the activities established for the profession and bureaucratic work. Agents from the municipality with less dedication to the bureaucracy reported a greater role in community mobilization and guidance on child health. The performance of ACS can be impaired if involved in bureaucratic tasks.

Key words: primary health care; community development; Community Health Workers.

Résumé: Cette étude visait à décrire le profil et la réalité du travail de l'agent de santé communautaire (ACS) dans deux municipalités de Paraiba. Etude transversale avec 98 ACS. La plupart des ACS a développé les activités établies pour la profession et de la paperasserie. Agents de comté avec moins de dévouement à la bureaucratie ont rapporté plus grand rôle dans la mobilisation des communautés et des conseils sur la santé des enfants. La performance ACS peut être affectée par la participation à la paperasserie.

Mots-clés: soins de sante primaires; le developpement communautaire; les Agents de Santé Communautaire. Resumen: Este estudio tuvo como objetivo describir el perfil y la realidad del trabajo del Agente Comunitario de Salud (ACS) en dos municipios de Paraíba. Estudio transversal con 98 ACS. La mayoría de los ACS desarrollaron las actividades establecidas para la profesión y trabajo burocrático. Agentes del municipio con menos dedicación a la burocracia trabajaban más en movilización comunitaria y orientación sobre la salud de los niños. El rendimiento de ACS puede afectarse al hacer trabajo burocrático.
\end{abstract}

Palabras clave: atención primaria de salud; desarrollo comunitario; Agentes Comunitarios de Salud.

\section{INTRODUÇÃO}

A Estratégia Saúde da Família (ESF) busca reorientar o modelo de atenção à saúde do Sistema Único de Saúde (SUS) por meio da aproximação dos profissionais de saúde à comunidade (JESUS et al., 2014). O Agente Comunitário de Saúde (ACS) apresenta-se, nessa conjuntura, com papel de destaque na equipe multiprofissional, uma vez que atua como elo entre equipe e

\footnotetext{
${ }^{1}$ Universidade Estadual da Paraíba (UEPB), Campina Grande, Paraíba, Brasil.

${ }^{2}$ Universidade Federal de Pelotas (UFPel), Pelotas, Rio Grande do Sul, Brasil.
} 
usuário (CARDOSO; NASCIMENTO, 2010; FILGUEIRAS; SILVA, 2011). A atuação desse profissional é fundamental para a reorganização dos serviços de saúde e a qualidade da assistência, uma vez que tem a importante função de integrar a comunidade aos serviços de saúde e vice-versa (CARDOSO; NASCIMENTO, 2010). Esse processo é facilitado na medida em que o agente mora na comunidade, está em contato permanente com as famílias e, simultaneamente, integra a equipe de saúde (CARDOSO; NASCIMENTO, 2010; PERES et al., 2011). Ainda, privilegia-se por possuir o saber técnico e popular em saúde (COSTA; CARVALHO, 2012).

Essas características apresentam significados importantes para o processo de trabalho do ACS, tornando-o complexo. Esse profissional recebe diretamente as queixas da população, comprometendo-se, de forma direta, com a necessidade de dar respostas e, ao mesmo tempo, deve confrontar-se com a equipe, tendo que agir segundo as possibilidades e os limites desta, bem como do próprio sistema de saúde (PERES et al., 2011).

Ainda, o ACS encontra-se inserido em um espaço de indefinições relacionadas às suas competências, habilidades e saberes (GALAVOTE et al., 2011), bem como de práticas que limitam sua atuação na mudança do modelo de saúde (GOMES et al., 2010). Considerando que o conhecimento sobre o processo de trabalho do ACS é oportuno e necessário para a qualificação do seu trabalho e a mudança de modelo proposta no seio da ESF na perspectiva da atenção centrada na saúde (BORNSTEIN; DAVID, 2014; COSTA et al., 2013), o objetivo deste estudo foi descrever o perfil e as atividades de trabalho desses profissionais em dois municípios do Estado da Paraíba, no sentido de contribuir para a consolidação da ESF.

\section{MÉTODOS}

Estudo de delineamento transversal, de abordagem quantitativa, que comparou o perfil e o processo de trabalho de ACS de equipes da ESF de dois municípios do Estado da Paraíba. Os municípios foram escolhidos considerando suas semelhanças em aspectos como a posição geográfica (localização na região metropolitana da capital do Estado com acesso à rede de serviços nela disponibilizados), grau de urbanização (de quase 100\%), indicadores demográfico-sociais (índice de desenvolvimento humano de nível médio- 0,649 no Município 1 e 0,748 no Município 2) e tradição na organização de serviços primários de saúde (cobertura populacional da ESF de quase 100\%). O Município 1 tem população de 99.716 habitantes, dos quais 7.862 são crianças menores de cinco anos, e possui sistema de saúde composto por 28 equipes da ESF. O Município 2 tem população de 57.944 habitantes, dos quais 4.596 são crianças menores de cinco anos, e possui sistema de saúde composto por 19 equipes da ESF. Entretanto, no Município 2, é maior o número de profissionais de saúde (médicos, pediatras, enfermeiros, nutricionistas), para cada 1.000 habitantes, no SUS, e as equipes da ESF são ampliadas com a inserção do nutricionista.

Em cada município, foram selecionadas, por sorteio aleatório simples, nove equipes da ESF (representando $1 / 3$ das equipes do Município 1 e cerca da metade das equipes do Município 2). Em cada equipe de saúde sorteada, o estudo incluiu, no mínimo, 1/3 dos ACS em atividade. No final do período de coleta de dados, a amostra ficou constituída por 98 ACS, sendo 54 do Município 1 e 44 do Município 2.

A coleta de dados foi realizada nas unidades de saúde entre julho e dezembro de 2014. A equipe de campo foi formada por profissionais e estudantes da área de saúde, com experiência prévia em trabalho de campo, o qual foi supervisionado por profissional capacitado. O controle de qualidade do estudo incluiu: treinamento e padronização dos entrevistadores, adequação 
do instrumento de coleta, construção de Manual de Instruções e realização de estudo piloto no município de Campina Grande, Paraíba.

Para a obtenção dos dados, foi utilizado questionário estruturado com questões fechadas que abordavam características pessoais, do trabalho e atividades desenvolvidas pelos ACS. As características pessoais referiram-se a sexo, idade, escolaridade, convivência com companheiro(a) e tempo de moradia na comunidade. As características do trabalho contemplaram informações sobre o tempo de trabalho na equipe de saúde e o número de famílias atendidas.

As atividades desempenhadas foram definidas com base nas atribuições definidas pelo Ministério da Saúde (Brasil, 2012), contemplando: visita domiciliar, educação em saúde, identificação e acompanhamento de grupos de risco, cadastramento das famílias, busca ativa de faltosos, promoção da mobilização e participação comunitária. Além disso, considerou-se a atuação no controle vacinal e em trabalho burocrático. Para descrever as ações de promoção da saúde e prevenção de agravos, o questionário também abordou o desenvolvimento de orientações às mães sobre aspectos relacionados à saúde das crianças. As perguntas foram extraídas do Instrumento de Avaliação da Atenção Primária (PCATool- Primary Care Assessment Tool), versão profissionais, componente integralidade- serviços prestados (BRASIL, 2010). Para os efeitos do presente trabalho, as categorias com alternativas "com certeza sim" e "provavelmente sim" foram agrupadas e consideradas como resposta positiva, enquanto as alternativas "com certeza não" e "provavelmente não" foram agrupadas e consideradas como resposta negativa.

Os dados coletados foram organizados em planilhas eletrônicas e digitados em dupla entrada. O aplicativo Validate do software Epi Info versão 3.3.2 foi usado para analisar a consistência dos dados gerando o banco final que foi utilizado nas análises estatísticas.

Realizou-se a análise descritiva dos dados em frequência absoluta e relativa, apresentados na forma de tabela. $O$ teste de $X^{2}$ foi utilizado para analisar diferenças entre os municípios em relação ao perfil e às condutas adotadas pelos ACS. Utilizou-se o software Stata versão 12.0, e o nível de significância admitido foi de $5 \%$.

A pesquisa foi aprovada pelo Comitê de Ética em Pesquisa da Universidade Estadual da Paraíba em 17/12/2013, com o protocolo de número 19689613.3.0000.5187. Todos os participantes da pesquisa assinaram o Termo de Consentimento Livre e Esclarecido como condição necessária e concordância relacionada à participação no estudo. A assinatura do Termo foi realizada após esclarecimentos necessários sobre as características da pesquisa. Todos os sujeitos tiveram respeitados os princípios de autonomia, não maleficência, beneficência, justiça e equidade.

\section{RESULTADOS}

Dos 98 ACS considerados no estudo, observa-se, segundo os dados da Tabela 1, que os profissionais eram na sua maioria mulheres, em maior proporção no Município 2 (93,18\%) quando comparado ao Município 1 (74,07\%). Predominaram ACS de faixa etária entre 30 e 39 anos $(55,10 \%)$, escolaridades $>=12$ anos de estudo, com companheiro(a) (64,29\%) e com 2 a 3 filhos $(44,90 \%)$, sem diferença estatística entre os municípios. Em relação ao tempo de moradia na região, 77,55\% eram moradores há mais de 20 anos na comunidade em que trabalham, sendo essa proporção estatisticamente maior no Município 2. Quase 90,0\% dos ACS trabalhavam na equipe de saúde fazia mais de dois anos. O número de famílias atendidas que predominou foi o de 120 a 169, em ambos os municípios. 
Tabela 1- Perfil e características do trabalho de agentes comunitários de saúde da Estratégia Saúde da Família de dois municípios do Estado da Paraíba, 2014.

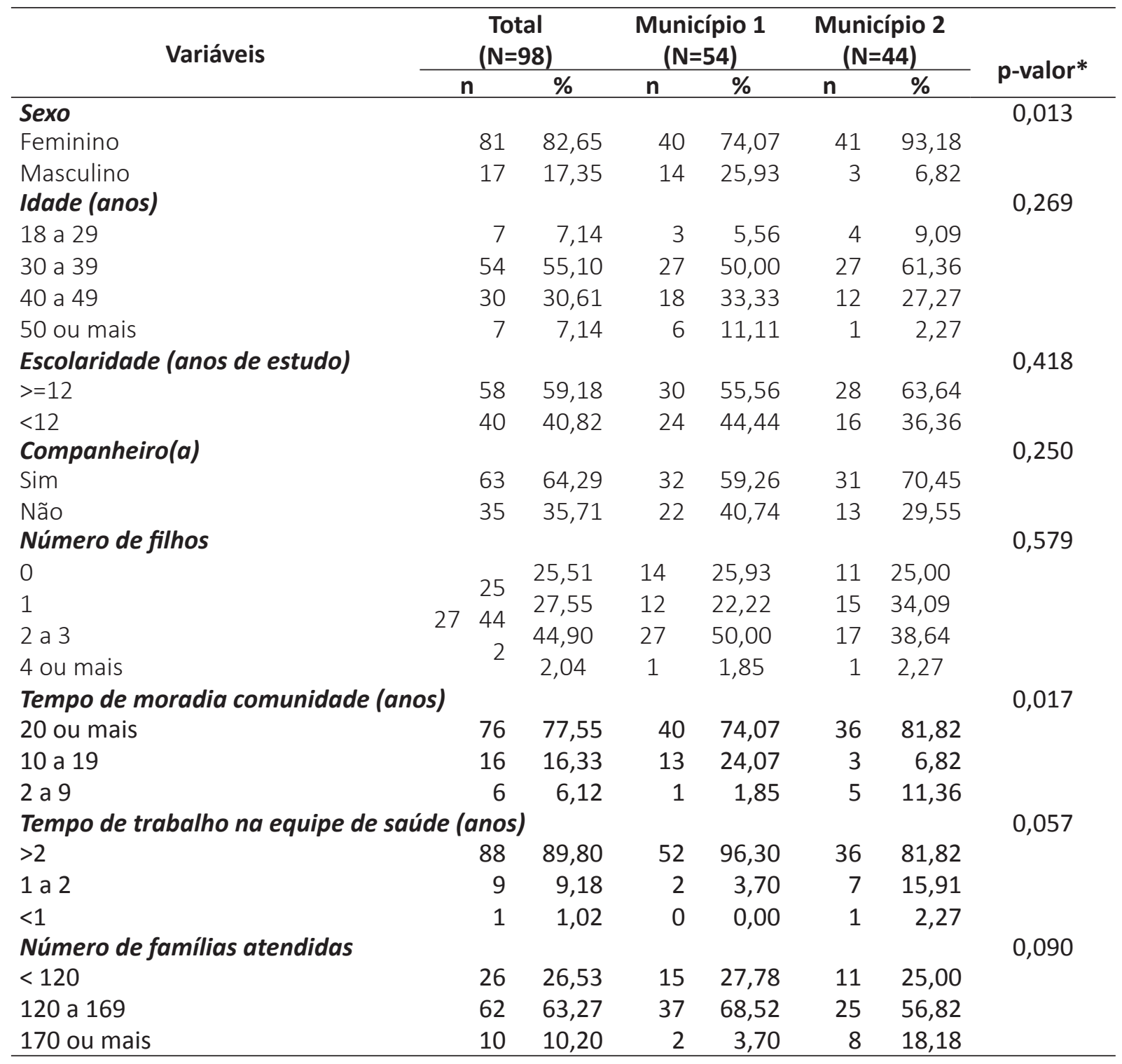

* Teste de $\mathrm{X}^{2}$.

Em relação às atividades desenvolvidas, observa-se que a visita domiciliar, o controle vacinal e o cadastramento eram praticados por todos os ACS. Todas as outras atividades eram realizadas pela maioria dos profissionais, com proporções que variaram de 78,57\% (atividades burocráticas) a 98,98\% (educação em saúde, busca ativa de faltosos e orientações sobre como manter as crianças saudáveis). Promoção da mobilização e participação comunitária, trabalho burocrático e orientações às mães sobre segurança no lar, respeito aos medicamentos, em relação aos problemas de comportamento e relacionadas ao crescimento e desenvolvimento das crianças foram atividades referidas mais frequentemente pelos ACS do Município 1, quando comparados aos do Município 2 (Tabela 2). 
Tabela 2- Atividades desenvolvidas por agentes comunitários de saúde da Estratégia Saúde da Família de dois municípios do Estado da Paraíba, 2014.

\begin{tabular}{|c|c|c|c|c|c|c|c|}
\hline \multirow[t]{2}{*}{ Atividades desenvolvidas } & \multicolumn{2}{|c|}{$\begin{array}{l}\text { Total } \\
(\mathrm{N}=98)\end{array}$} & \multicolumn{2}{|c|}{$\begin{array}{l}\text { Município } 1 \\
\quad(\mathrm{~N}=54)\end{array}$} & \multicolumn{2}{|c|}{$\begin{array}{c}\text { Município } 2 \\
(\mathrm{~N}=44)\end{array}$} & \multirow{2}{*}{ p-valor* } \\
\hline & $\mathbf{n}$ & $\%$ & $\mathbf{n}$ & $\%$ & $\mathbf{n}$ & $\%$ & \\
\hline Visita domiciliar & 98 & 100,0 & 54 & 100,0 & 44 & 100,0 & 1,00 \\
\hline Educação em saúde & 97 & 98,98 & 54 & 100,0 & 43 & 97,73 & 0,265 \\
\hline $\begin{array}{l}\text { Identificaçao e acompanhamento de } \\
\text { grupos de risco }\end{array}$ & 94 & 95,92 & 52 & 96,30 & 42 & 95,45 & 0,834 \\
\hline Controle vacinal & 98 & 100,0 & 54 & 100,0 & 44 & 100,0 & 1,00 \\
\hline Cadastramento das famílias & 98 & 100,0 & 54 & 100,0 & 44 & 100,0 & 1,00 \\
\hline Busca ativa de faltosos & 97 & 98,98 & 53 & 98,15 & 44 & 100,0 & 0,364 \\
\hline $\begin{array}{l}\text { Promoção da mobilização e participação } \\
\text { comunitária }\end{array}$ & 88 & 89,80 & 54 & 100,0 & 34 & 77,27 & 0,000 \\
\hline Trabalho burocrático & 77 & 78,57 & 38 & 70,37 & 39 & 88,64 & 0,028 \\
\hline $\begin{array}{l}\text { Orientações sobre como manter as } \\
\text { crianças saudáveis }\end{array}$ & 97 & 98,98 & 54 & 100,0 & 43 & 97,73 & 0,265 \\
\hline $\begin{array}{l}\text { Orientações sobre segurança no lar com } \\
\text { medicamentos }\end{array}$ & 85 & 86,73 & 51 & 94,44 & 34 & 77,27 & 0,013 \\
\hline $\begin{array}{l}\text { Orientações sobre como manter as } \\
\text { crianças seguras }\end{array}$ & 90 & 91,84 & 51 & 94,44 & 39 & 88,64 & 0,296 \\
\hline $\begin{array}{l}\text { Orientações sobre o comportamento das } \\
\text { crianças }\end{array}$ & 82 & 83,67 & 49 & 90,74 & 33 & 75,00 & 0,036 \\
\hline $\begin{array}{l}\text { Orientações sobre crescimento e } \\
\text { desenvolvimento das crianças }\end{array}$ & 88 & 89,80 & 53 & 98,15 & 35 & 79,55 & 0,002 \\
\hline
\end{tabular}

* Teste de $\mathrm{X}^{2}$.

\section{DISCUSSÃO}

A maioria dos ACS pesquisados neste estudo era do sexo feminino, assim como em outros municípios brasileiros (FREITAS et al., 2015; GALAVOTE et al., 2011; GARCIA et al., 2017; SALIBA et al., 2011). Esse fato pode estar ligado ao papel da mulher no cuidado da saúde (FREITAS et al., 2015) ou à liderança assumida associada à busca de crescimento profissional (SALIBA et al., 2011). Historicamente, a mulher tem sido vista como cuidadora, o que lhe confere credibilidade e sensibilidade com a comunidade (GALAVOTE et al., 2011; GARCIA et al., 2017) e pode ter contribuído na sua predominância como trabalhadora e usuária mais frequente dos serviços de saúde (FREITAS et al., 2015; SALIBA et al., 2011).

Foi constatado que todos os ACS encontraram-se dentro da faixa etária estabelecida (BRASIL, 2001), cujos resultados são semelhantes aos de pesquisas anteriores (FERRAZ; AERTS, 2005; FREITAS et al., 2015; GARCIA et al., 2017). Discute-se que ACS com mais idade, como neste estudo, propicia melhor conhecimento da comunidade e vínculo com ela. (FERRAZ; AERTS, 2005).

Quanto à família, constatou-se, similarmente a outros estudos (FERRAZ; AERTS, 2005; FREITAS et al., 2015), que a maioria dos agentes viviam com companheiro(a) e tinham filhos, indicadores esses que podem atestar, também, o cumprimento da exigência relacionada à permanência de moradia na microárea onde trabalha (BRASIL, 2001). A vivência da maternidade pode representar uma experiência positiva de aprendizado e habilidades no contexto dos cuidados à saúde infantil, os quais devem ser aprimorados por meio da educação permanente e supervisão das atividades desenvolvidas pelo agente (FERRAZ; AERTS, 2005). 
Considerando que o Ministério da Saúde estabeleceu como critério de escolaridade para exercer a função de ACS apenas as habilidades de ler e escrever (BRASIL, 2001), 100\% dos profissionais deste estudo cumpriam o requisito. Minimizar a escolaridade a essa exigência pode prejudicar a incorporação de novos conhecimentos condicionados a maior nível escolar (SALIBA et al., 2011). Ainda, esse requisito contraria a responsabilidade do ACS na mudança do paradigma biomédico, que, por sua vez, permeia a formação e as práticas desse profissional (FONSECA et al., 2012; FONSECA; MENDONÇA, 2014), sendo um dos principais desafios associados à reorientação do sistema (RIGON; SCHMIDT; BÓGUS, 2016). Cabe destacar que outros referenciais estabelecem a conclusão do ensino fundamental (BRASIL, 2006) ou de curso técnico (BRASIL, 2004) para o exercício da função. Apesar de referenciais discordantes, estabelece-se a preocupação com a elevação da escolaridade desse profissional (BORNSTEIN; DAVID, 2014; QUEIRÓS; LIMA, 2012). Experiências de formação técnica sugerem sua importância na aquisição de conhecimentos e qualificação do processo de trabalho do ACS, bem como para a própria vida do indivíduo (BORNSTEIN; DAVID, 2014).

A maioria dos agentes morava na mesma comunidade em que trabalhava por período de 10 anos ou mais, e todos por tempo mínimo de dois anos, o que satisfaz às normas emanadas pelo Ministério da Saúde (BRASIL, 2001). Resultado similar foi encontrado por outros pesquisadores (FERRAZ; AERTS, 2005; SALIBA et al., 2011). A residência do agente na mesma comunidade onde trabalha considera-se um fator facilitador das funções na ESF (QUEIRÓS; LIMA, 2012; MENEGUSSI; OGATA; ROSALINI, 2014; SALIBA et al., 2011), o que, no entanto, pode ser fonte de desgaste emocional para esse trabalhador (MENEGUSSI; OGATA; ROSALINI, 2014). É importante ressaltar que, apesar de o tempo de dois anos ser considerado condição necessária para que o agente conheça a comunidade e com ela crie vínculos (COSTA; FERREIRA, 2012; FERRAZ; AERTS, 2005; SALIBA et al., 2011), isso não garante a qualidade da relação entre eles, uma vez que esse tipo de vínculo tem influência de múltiplos fatores, a exemplo da dupla identidade do agente (como trabalhador e membro da comunidade), a motivação e a satisfação com o trabalho (COSTA; FERREIRA, 2012; MENEGUSSI; OGATA; ROSALINI, 2014; QUEIRÓS; LIMA, 2012; SALIBA et al., 2011).

Os ACS deste estudo atendiam, predominantemente, entre 170 a 219 famílias, o que corresponde à faixa na qual se inclui a média de famílias por profissional preconizada (FERRAZ; AERTS, 2005). A importância de os ACS não terem famílias em excesso na sua microárea de atuação está associada à garantia da realização de visitas a todas as famílias mensalmente, bem como do número de horas dispensadas para essa atividade (FERRAZ; AERTS, 2005). A adequação na formação e no vínculo de trabalho do ACS é indispensável à melhoria da cobertura dos serviços de saúde no contexto do SUS (VIEIRA, 2011).

A realização de visitas domiciliares, a educação em saúde, o cadastramento de famílias nas áreas de abrangência da ESF, a identificação e acompanhamento de grupos de risco, a busca ativa, a mobilização comunitária e a orientação sobre saúde aos usuários são atribuições do ACS (BRASIL, 2012) que foram devidamente atendidas pela maioria dos profissionais que fizeram parte deste estudo. Esses resultados mostram consistência com a literatura que destaca ações, a exemplo da visita domiciliar e da educação em saúde, como atividades básicas do cotidiano e efetivamente desenvolvidas pelo ACS, bem como revela a importância desses profissionais com a promoção da saúde (BRITO; FERREIRA; SANTOS, 2014; FREITAS et al., 2015; GALAVOTE et al., 2011; MACHADO et al., 2015; PEREIRA; OLIVEIRA, 2013; SALIBA et al., 2011), o que pode contribuir positivamente na reorientação do modelo assistencial (FONSECA et al., 2012; FONSECA; 
MENDONÇA, 2014; PEREIRA; OLIVEIRA, 2013). O papel mediador do agente possibilita que as pessoas se apropriem e aceitem as políticas de saúde, ampliando a visão da saúde na perspectiva da promoção (LOTTA; PAVEZ, 2010). Nesse contexto, a visita domiciliar gera vínculo, responsabilização, aumenta o acesso às informações e facilita a credibilidade do profissional por parte da população, criando melhores condições para a resolução das demandas de saúde (GALAVOTE et al., 2011; GARCIA et al., 2017; LOTTA; PAVEZ, 2010).

O envolvimento do ACS com atividades burocráticas, que descaracterizam suas funções e transcendem seus conhecimentos, com prejuízos à implantação de atividades próprias da profissão (BRITO; FERREIRA; SANTOS, 2014; FERRAZ; AERTS, 2005; FONSECA; MENDONÇA, 2014), sugere-se por meio dos resultados atuais, ao indicar melhor desempenho nas ações de mobilização comunitária e de promoção (orientações às mães) entre os profissionais do município com menor dedicação a trabalho burocrático. A desburocratização do trabalho do agente é importante para as conquistas na mudança de modelo de atenção pretendida pela ESF (GALAVOTE et al., 2011), se bem que pode potencializar a capacidade de adaptar as políticas ao cotidiano (LOTTA; PAVEZ, 2010). Em estudo realizado em Recife, PE, apontou-se, de forma semelhante, que a organização da comunidade tem perdido importância na atuação do ACS, associado à assunção de novas atribuições que dispensam tempo adicional (QUEIRÓS; LIMA, 2012). Resultados originados de investigação conduzida em vários municípios, por exemplo, apontou que os ACS atuavam em 1.080 práticas diferentes, das quais aproximadamente 64\% não estão previstas na legislação (LOTTA; PAVEZ, 2010). É essencial que o ACS possa contribuir com práticas de orientação sobre a saúde da criança, pois seu trabalho é valorizado como precursor da educação popular em saúde, ao situar-se entre saberes técnico e popular (MACIAZEKI-GOMES et al., 2016). Nessa perspectiva, cabe destacar a importância de existirem condições propícias nas equipes de saúde para que os saberes e práticas populares do ACS não contribuam negativamente nas relações desse profissional com os outros membros da equipe e na institucionalização do seu trabalho (BORNSTEIN; DAVID, 2014; QUEIRÓS; LIMA, 2012).

Podem-se reconhecer limitações neste estudo condicionadas à avaliação segundo respostas dos próprios profissionais, com possibilidade de informações enviesadas, e por tratar das condições apenas de dois municípios, o que restringe a generalização dos resultados encontrados. Entretanto a coleta dos dados foi padronizada, sem perdas e com alta aceitação por não haver recusas dos entrevistados. Além disso, a comparação de duas realidades revela-se importante na medida em que as pesquisas sobre o tema são geralmente focadas em uma localidade geográfica.

Conclui-se que o ACS desenvolve adequadamente as funções estabelecidas para a profissão. O ACS atua, também, em tarefas burocráticas que descaracterizam suas atribuições e podem prejudicar o seu desempenho. É importante que o agente não assuma tais responsabilidades de forma que sua atuação corresponda às expectativas sobre ele depositadas. Sugere-se a adoção de medidas que possam potencializar o trabalho do ACS como a inclusão de pessoal administrativo nas equipes de saúde, capacitação, educação permanente e sensibilização sobre a importância do desenvolvimento de ações de promoção da saúde e prevenção de agravos de forma adequada. 


\section{REFERÊNCIAS}

BORNSTEIN, Vera J.; DAVID, Helena M. S. L. Contribuições da formação técnica do agente comunitário de saúde para o desenvolvimento do trabalho da equipe saúde da família. Trabalho, Educação e Saúde, Rio de Janeiro, v. 12, n. 1, p. 107-28, 2014.

BRASIL. Ministério da Saúde. Política Nacional de Atenção Básica. Brasília, DF: Ministério da Saúde, 2012. (Série E. Legislação em Saúde).

Ministério da Saúde. Manual do instrumento de avaliação da atenção primária à saúde: primary care assessment tool pcatool - Brasil. Brasília: Ministério da Saúde, 2010.

. Lei n. 11.350, de 5 de outubro de 2006. Regulamenta o §5o do art. 198 da Constituição, dispõe sobre o aproveitamento de pessoal amparado pelo parágrafo único do art. 20 da Emenda Constitucional no 51, de 14 de fevereiro de 2006, e dá outras providências. Brasília, 2006.

Ministério da Saúde. Ministério da Educação. Referencial curricular para curso técnico de agentes comunitários de saúde: área profissional saúde. Brasília: Ministério da Saúde, 2004.

. Ministério da Saúde. Programa agentes comunitários de saúde (PACS). Brasília: Ministério da Saúde, 2001.

BRITO, Rosineide S.; FERREIRA, Nathaly E. M. S.; SANTOS, Danyelle L. A. Atividades dos Agentes Comunitários de Saúde no âmbito da Estratégia Saúde da Família: revisão integrativa da literatura. Saúde \& Transformação Social, Florianópolis, v. 5, n. 1, p. 16-21, 2014.

CARDOSO, Andréia S.; NASCIMENTO, Marilene C. Comunicação no Programa Saúde da Família: o agente de saúde como elo integrador entre a equipe e a comunidade. Ciência \& Saúde Coletiva, Rio de Janeiro, v. 15, supl. 1, p. 1509-20, 2010.

COSTA, Elaine M.; FERREIRA, Danyege L. A. Percepções e motivações de agentes comunitários de saúde sobre o processo de trabalho em Teresina, Piauí. Trabalho, Educação e Saúde, Rio de Janeiro, v. 9, n. 3, p. 461-78, 2012.

COSTA, Samira L.; CARVALHO, Emílio N. Agentes Comunitários de Saúde: agenciadores de encontros entre territórios. Ciência \& Saúde Coletiva, Rio de Janeiro, v. 17, n. 11, p. 2931-40.

COSTA, Simone M. et al. Agente Comunitário de Saúde: elemento nuclear das ações em saúde. Ciência \& Saúde Coletiva, Rio de Janeiro, v. 18, n. 7, p. 2147-56, 2013.

FERRAZ, Lucimare; AERTS, Denise R. G. C. O cotidiano de trabalho do agente comunitário de saúde no PSF em Porto Alegre. Ciência \& Saúde Coletiva, Rio de Janeiro, v. 10, n. 2, p. 347-55, 2005.

FILGUEIRAS, Andréa S.; SILVA, Ana L. A. Agente Comunitário de Saúde: um novo ator no cenário da saúde do Brasil. Physis, Rio de Janeiro, v. 21, n. 3, p. 899-915, 2011.

FONSECA, Angélica F.; MENDONÇA, Maria H. M. A interação entre avaliação e a atuação dos Agentes Comunitários de Saúde: subsídios para pensar sobre o trabalho educativo. Saúde em Debate, Rio de Janeiro, v. 38, especial, p. 343-57, 2014.

FONSECA, Angélica F. et al. Avaliação em saúde e repercussões no trabalho do agente comunitário de saúde. Texto Contexto e Enfermagem, Florianópolis, SC, v. 21, n. 3, p. 519-27, 2012.

FREITAS, Lagerson M. et al. Formação dos agentes comunitários de saúde no município de Altamira (PA), Brasil. ABCS Health Science, Santo André, SP, v. 40, n. 3, p. 171-7, 2015.

GALAVOTE, Heletícia S. et al. Desvendando os processos de trabalho do agente comunitário de saúde nos cenários revelados na Estratégia Saúde da Família no município de Vitória (ES, Brasil). Ciência \& Saúde Coletiva, Rio de Janeiro, v. 16, n. 1, p. 231-40, 2011.

GARCIA, Ana Claudia P. et al. Agente comunitário de saúde no Espírito Santo: do perfil às atividades desenvolvidas. Trabalho, Educação e Saúde, Rio de Janeiro, v. 15, n. 1, p. 283-300, 2017. 
GOMES, Karine O. et al. O Agente Comunitário de Saúde e a consolidação do Sistema Único de Saúde: reflexões contemporâneas. Physis, Rio de Janeiro, v. 20, n. 4, p. 1143-64, 2010.

JESUS, Andreia S. et al. Atuação do agente comunitário de saúde: conhecimento de usuários. Revista de Enfermagem UERJ, Rio de Janeiro, v. 22, n. 2, p. 239-44, 2014.

LOTTA, Gabriela S.; PAVEZ, Thais R. Agentes de implementação: mediação, dinâmicas e estruturas relacionais. Cadernos Gestão Pública e Cidadania, São Paulo, v. 15, n. 56, p. 109-125, 2010.

MACHADO, Letícia M. et al. Estratégia Saúde da Família: a percepção do agente comunitário de saúde quanto a sua atuação. Ciência, Cuidado e Saúde, Maringá, PR, v. 14, n. 2, p. 1105-12, 2015.

MACIAZEKI-GOMES, Rita C. et al. O trabalho do agente comunitário de saúde na perspectiva da educação popular em saúde: possibilidades e desafios. Ciência \& Saúde Coletiva, Rio de Janeiro, v. 21, n. 5, p. 1637-46, 2016.

MENEGUSSI, Juliana M.; OGATA, Márcia N.; ROSALINI, Maria H. P. O agente comunitário de saúde como morador, trabalhador e usuário em São Carlos, São Paulo. Trabalho, Educação e Saúde, Rio de Janeiro, v. 12, n. 1, p. 87-106, 2014.

PEREIRA, lara C.; OLIVEIRA, Maria A. C. O trabalho do agente comunitário na promoção da saúde: revisão integrativa da literatura. Revista Brasileira de Enfermagem, Brasília, v. 66, n. 3, p. 412-9, 2013.

PERES, Cássia R. F. B. et al. O agente comunitário de saúde frente ao processo de trabalho em equipe: facilidades e dificuldades. Revista da Escola de Enfermagem da USP, São Paulo, v. 45, n. 4, p. 905-11, 2011.

QUEIRÓS, Agleildes A. L.; LIMA, Luci P. A institucionalização do trabalho do agente comunitário de saúde. Trabalho, Educação e Saúde, Rio de Janeiro, v. 10, n. 2, p. 257-81, 2012.

RIGON, Silvia A.; SCHMIDT, Suely T.; BÓGUS, Cláudia M. Desafios da nutrição no Sistema Único de Saúde para construção da interface entre a saúde e a segurança alimentar e nutricional. Cadernos de Saúde Pública, Rio de Janeiro, v. 32, n. 3, e00164514, 2016.

SALIBA, Nemre A. et al. Agente comunitário de saúde: perfil e protagonismo na consolidação da atenção primária à saúde. Cadernos de Saúde Coletiva, Rio de Janeiro, v. 19, n. 3, p. 318-26, 2011.

VIEIRA, M. Para além da comunidade: trabalho e qualificação dos agentes comunitários de saúde. Rio de Janeiro: EPSJV, 2011.

\section{Sobre os autores:}

Dixis Figueroa Pedraza: Bacharel em Alimentos pela Universidad de La Habana, Mestre em Nutrição em Saúde Pública pelo Instituto Superior de Ciencias Médicas de La Habana, Doutor em Nutrição pela Universidade Federal de Pernambuco (UFPE) e Pós-doutor em Epidemiologia pela Universidade Federal de Pelotas (UFPel). Professor Doutor do Departamento de Enfermagem e Programa de Pós-Graduação em Saúde Pública da Universidade Estadual da Paraíba (UEPB). E-mail: dixisfigueroa@gmail.com

Iná Santos: Graduada em Medicina pela Faculdade de Medicina da Universidade Federal de Pelotas (UFPel), fez residência em Medicina Geral Comunitária no Hospital Nossa Senhora Conceição e especialização em Saúde Pública na Escola de Saúde Pública da Secretaria de Saúde e Meio Ambiente do Estado do Rio Grande do Sul. Mestre e doutora em Ciências Médicas, com área de concentração em Epidemiologia, pela Universidade Federal do Rio Grande do Sul (UFRGS).

E-mail: inasantos@uol.com.br 
